\title{
Identifying global RNA-chromatin interactions by GRID-seq
}

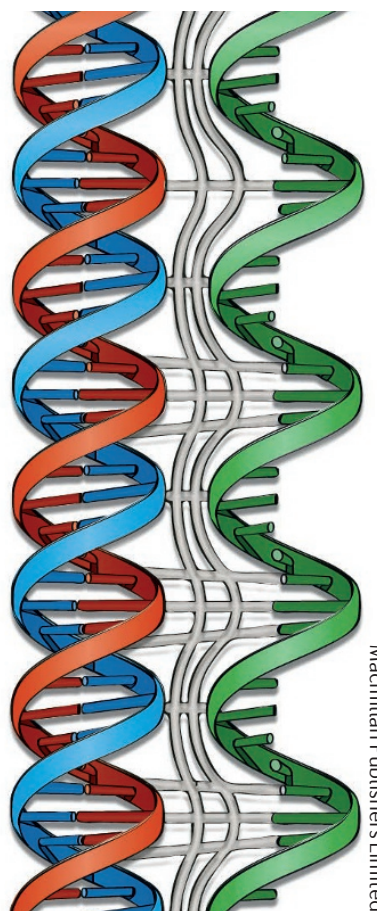

A number of techniques can detect interactions between RNA molecules and chromatin, but these techniques only capture chromatin interactions of individual RNAs. Now, Li et al. describe a new method that maps global RNA interactions with DNA by deep sequencing (GRID-seq) and promises to provide an unbiased identification of all potential chromatin-interacting RNAs.

In this method, a bivalent linker comprising single-stranded RNA and double-stranded DNA is used to link RNA to DNA in fixed nuclei, followed by amplification and deep sequencing to detect the RNA-DNA interactions. The method was validated by showing that the GRIDseq interaction data for a single RNA, the long non-coding RNA MALAT1, in a mouse embryonic stem (ES) cell line is comparable to 람 that obtained using an established non-global technique, RNA affinity purification followed by sequencing. GRID-seq analysis of the human MDA-MD-231 breast cancer cell line revealed that most RNA reads originated from partially spliced RNAs, whereas most DNA reads were from within promoters and intergenic regions. In addition to MDA-MD-231 cells and mouse ES cells, GRID-seq analysis of the human MM.1S multiple myeloma cell line and of Drosophila melanogaster $\mathrm{S} 2$ cells revealed that distinct classes of RNAs mediate cischromosome and trans-chromosome interactions; this phenomenon is probably linked to cell typespecific patterns of gene expression. However, most RNAs interacted with cis-chromosome locations that are close to their sites of transcription. This interaction distance was much smaller in S2 cells, reflecting the more compact gene organization in D. melanogaster compared with mammals.

GRID-seq may also provide information about the strength of enhancers, as chromatininteracting RNAs are more abundant at super-enhancers (enhancers enriched for transcriptional regulator binding or chromatin marks) than at typical enhancers. Whereas individual chromatin-enriched RNAs interacted with multiple typical enhancers but only one or two super-enhancers, most enhancers only interacted with RNAs from one or two genes, implying that each gene is regulated by multiple enhancers, whereas each enhancer seems to regulate a selective set of genes.

As many unannotated chromatinenriched RNAs were detected by GRID-seq analysis in all cell types examined, this method will provide many RNA-chromatin interactions for future study.

Grant Otto

ORIGINAL ARTICLE Li, X. et al. GRID-seq reveals the global RNA-chromatin interactome. Nat. Biotechnol. http://dx.doi.org/10.1038/nbt.3968 (2017) 